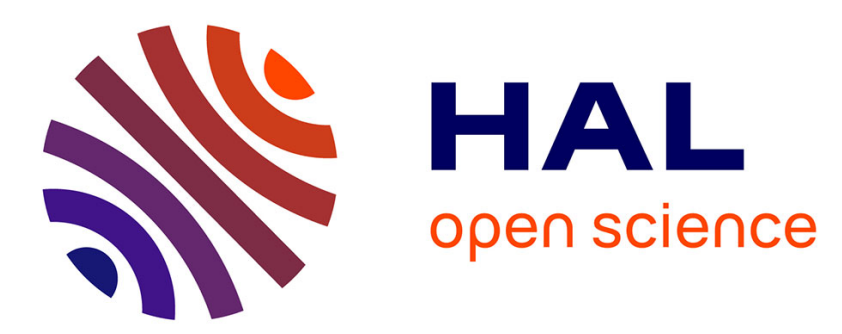

\title{
Composition fluctuations in polydisperse liquids: Glasslike effects well above the glass transition
}

L. Klochko, J. Baschnagel, J. P Wittmer, O. Benzerara, C. Ruscher, Alexander N Semenov

\section{- To cite this version:}

L. Klochko, J. Baschnagel, J. P Wittmer, O. Benzerara, C. Ruscher, et al.. Composition fluctuations in polydisperse liquids: Glasslike effects well above the glass transition. Physical Review E , 2020, 102 (4), 10.1103/PhysRevE.102.042611 . hal-03255091

\section{HAL Id: hal-03255091 https://hal.science/hal-03255091}

Submitted on 9 Jun 2021

HAL is a multi-disciplinary open access archive for the deposit and dissemination of scientific research documents, whether they are published or not. The documents may come from teaching and research institutions in France or abroad, or from public or private research centers.
L'archive ouverte pluridisciplinaire HAL, est destinée au dépôt et à la diffusion de documents scientifiques de niveau recherche, publiés ou non, émanant des établissements d'enseignement et de recherche français ou étrangers, des laboratoires publics ou privés. 


\title{
Composition fluctuations in polydisperse liquids: glass-like effects well above the glass transition
}

\author{
L.Klochko, J.Baschnagel, J.P. Wittmer, O.Benzerara, C. Ruscher, A.N.Semenov* \\ Institut Charles Sadron, CNRS - UPR 22, Université de Strasbourg, \\ 23 rue du Loess, BP 84047, 67034 Strasbourg Cedex 2, France
}

(Dated: September 25, 2020)

\begin{abstract}
We study a 2-dimensional glassforming system of slightly polydisperse LJ particles using MD simulations, and demonstrate that in the liquid regime (well above the vitrification temperature) this model shows a number of features typical of the glass transition: (i) the relation between compressibility and structure factor $S(q)$ is strongly violated; (ii) the dynamical structure factor $S(q, t)$ at low $q$ shows a two-step relaxation; (iii) the time-dependent heat capacity $c_{v}(t)$ shows a long-time power-law tail. We show that these phenomena can be rationalized with the idea of composition fluctuations and provide a quantitative theory for the effects (i) and (ii). It implies that such effects must be inherent in all polydisperse colloidal models including binary LJ mixtures.
\end{abstract}

PACS numbers: 05.20.Jj,64.70.kj

\section{INTRODUCTION}

It is well-known that vitrification of molecular liquids or polymer melts is accompanied by a number of characteristic features $[1,2]$. One of these features is the emergence of an intermediate plateau in dynamical correlation functions, like the shear relaxation modulus $G(t)$ or the dynamical structure factor $S(q, t)$ [3], upon cooling the liquid toward the glass-transition temperature $T_{\mathrm{g}}$. The plateau is truncated by the (segmental) $\alpha$-process. As the associated relaxation time $\tau_{\alpha}$ grows with decreasing temperature $(T)$, the plateau increases in size, turning into a finite long-time plateau for $T<T_{\mathrm{g}}$ on the experimental time scale $\Delta t$ since $\tau_{\alpha} \gg \Delta t$. Another feature of the glass transition is the emergence of nonequilibrium effects for $T \lesssim T_{\mathrm{g}}$. One example is the drop of the specific heat $\left(c_{p}\right.$ or $\left.c_{v}\right)$ on cooling through $T_{\mathrm{g}}$ [4]. In the liquid phase, this effect is supplemented by a significant frequency dependence of the dynamic heat capacity implying its slow increase toward the equilibrium value on long time scales [5,6]. Another example is the violation of the relation between the compressibility and the structure factor $S(0) \equiv S(q \rightarrow 0)$ for vanishing wave vector $\underline{q}$ in the glass. The latter relation - the so-called compressibility equation $(\mathrm{CE})$ - is given by $[3,7]$

$$
S(0)=\frac{c_{0} T}{K_{T}}
$$

where $K_{T}$ is the static isothermal bulk compression modulus and $c_{0}=N / V$, the mean concentration of particles [8]. This fluctuation-dissipation relation is valid in the equilibrium liquid, but is not satisfied below $T_{\mathrm{g}}[2,9,10]$, where the concept of an effective 'compressibility temperature' $T_{\chi}$ defined via eq. 1 was introduced ( $T_{\chi}$ is higher than the actual temperature $T$ for $\left.T<T_{\mathrm{g}}\right)[10]$.

Many glass-forming systems have multiple components to suppress the tendency for structural ordering [11-14]. For systems with $n$ components it is known since the work by Kirkwood and Buff that the CE must be modified even under equilibrium conditions $[3,15,16]$. The Kirkwood-Buff theory expresses the compressibility in terms of the inverse matrix of partial structure factors $S_{i j}(q \rightarrow 0)$ where $i, j=1, \ldots n$. For binary systems, the resulting expression for $S(0)$ takes a compact form: $S(0)$ is given as a sum of $c_{0} T / K_{T}$ and a term related to composition fluctuations and their coupling to number fluctuations $[8,17]$. This expression can be utilized to analyze experimental data [17]. However, if $n$ is large, the matrix inversion becomes "conceptually and computationally difficult" [18], in particular in the limit of continuous distributions of particle sizes. To cope with this problem, a systematic expansion of the Kirkwood-Buff theory in terms of the size deviation from the mean particle diameter was suggested [18]. This method is powerful if structural information about partial pair correlations is available, as demonstrated by applications to jammed packings of size-disperse spheres [18] and random close-packed colloidal dispersions [19].

Here we propose a different approach that does not require knowledge on partial pair correlations, but combines thermodynamic $\left(K_{T}\right)$ and simple compositional information (related to the polydispersity index of particle sizes, $\left.\mathrm{PDI}=\delta_{\mathrm{p}}\right)$ to predict the polydispersity contribution $\left(S_{\mathrm{pl}}\right)$ to static, $S(q)$, and dynamical, $S(q, t)$, structure factors for low $q$. Our approach is valid in any spacial dimension $(d)$ and explains why the monodisperse CE, 1 , can be violated strongly even for systems with very low PDI $\left(\delta_{\mathrm{p}} \sim 1 \%\right)$. We compare our theory to results from molecular dynamics (MD) simulations of a two-dimensional (2D) system of polydisperse particles and show that polydispersity gives rise to glass-like effects already for $T$ well above $T_{\mathrm{g}}$ : $S(q, t)$ at low $q$ decays in 2 steps with an intermediate plateau (at $S \approx S_{\mathrm{pl}}$ ), and the time-dependent specific heat increases slowly toward its static equilibrium value (with 
a power-law relaxation for the gap). These relaxation features persist upon cooling toward $T_{\mathrm{g}}$ and are expected to interplay with the glassy dynamics [20-23].

In the next section we describe the model and the computational approach. Then we turn to the numerical results for the static structure factor (section III A) and dynamical correlation functions (section III B). The theory of the long-time correlation effects is presented in section IV. The paper is summarized in the last section V.

\section{MODEL AND NUMERICAL ALGORITHM}

\section{A. Model}

We study a 2-dimensional (2D) polydisperse system of Lennard-Jones (LJ) particles [27, 31]. The total number of particles is $N=10^{4}$. Similar models, including Kob-Andersen binary mixtures [32], are widely used in simulations of (2D and 3D) glass-forming liquids [12]. The diameters of the particles, $\sigma_{l}$ with $l=1 . . N$, are uniformly distributed between $(1-\Delta) \bar{\sigma}$ and $(1+\Delta) \bar{\sigma}$ with $\Delta=0.2$. The mean-square particle size is $\overline{\sigma^{2}}=\left(1+\Delta^{2} / 3\right) \bar{\sigma}^{2}$, leading to the size polydispersity index of $\delta_{\mathrm{p}}=\overline{\sigma^{2}} / \bar{\sigma}^{2}-1=\Delta^{2} / 3 \approx 0.013$. All particles have the same mass $m_{p}$. Following Refs. $[27,31]$, each pair of particles (of diameters $\sigma_{l}$ and $\sigma_{k}$ ) interacts with energy $u_{\mathrm{LJ}}\left(r / \sigma_{l k}\right)$, where $\sigma_{l k}=\left(\sigma_{l}+\sigma_{k}\right) / 2$ and $u_{\mathrm{LJ}}(s)=4 \varepsilon\left(s^{-12}-s^{-6}\right)$. The energy is truncated at $s_{\text {cut }}=2^{7 / 6} \approx 2.24$ and shifted to avoid discontinuity, so that $u(s)=0$ for $s>s_{\text {cut }}$. In what follows, all quantities are given in LJ units, i.e., the energy $\varepsilon$, the particle mass $m_{p}$, the mean particle diameter $\bar{\sigma}$ and the Boltzmann constant $k_{\mathrm{B}}$ are set to unity. This also implies that the Lennard-Jones time $\tau_{\mathrm{LJ}}=\sqrt{m_{p} \bar{\sigma}^{2} / \varepsilon}=1$. Standard periodic boundary conditions (PBC) were applied. The system was kept at constant external pressure $p_{0}=2$. The linear dimension of the simulation box, $L \sim 100$, thus shows a weak decrease with temperature $T$. The system volume $V=L^{d}, d=2$, so that $V \sim N$ and particle concentration $c_{0}=N / V \sim 1$.

\section{B. Equilibration and simulation runs}

The particle swap Monte-Carlo (MC) technique [12], combined with volume-fluctuation moves to impose a constant pressure, was used to equilibrate the system. The MC dynamics was employed only at the tempering stage to accelerate the structural relaxation and to prepare $m=50 \div 100$ well-equilibrated independent configurations. The so-obtained independent configurations served as starting points for molecular dynamics (MD) simulations (using the LAMMPS code [26]). Each configuration was first tempered for $\Delta t_{1}=2 \cdot 10^{5}$ in the NPT ensemble (using the Nosé-Hoover thermostat and barostat) to further equilibrate the system state including the particle velocities and then for another $\Delta t_{1}$ in the canonical NVT ensemble (using Nosé-Hoover thermostat) where the volume $V$ was fixed at a volume corresponding to $p_{0}=2$. The production runs (served to obtain all correlation functions) were performed during the sampling time $\Delta t=10^{5}$ in the NVT ensemble.

Parallel to that, we also carried out cooling runs using MD only. The cooling runs consisted of two steps: continuous cooling from the initial temperature $T=1$ with rate $-\mathrm{d} T / \mathrm{d} t=10^{-5}$ followed by NPT and NVT tempering as described above. These cooling runs allowed to determine the glass transition temperature $T_{\mathrm{g}}$. $T_{\mathrm{g}}$ depends on the total time spent during cooling and tempering. For the times on the order of $10^{5}$ used in this study we obtained $T_{\mathrm{g}} \approx 0.26$ defining $T_{\mathrm{g}}$ as the onset of a quasi-static elasticity (when the long-time shear modulus $\mu$ exceeds $1 \%$ of the instantaneous affine shear modulus $\mu_{\mathrm{A}}$ ). Nearly the same $T_{\mathrm{g}}$ was obtained previously from $\mathrm{MC}$ simulations using a similar continuous cooling protocol and a dilatometric criterion [31].

For $T>T \mathrm{~g}$ equilibration can also be achieved by MD. The results obtained after tempering by SWAP Monte Carlo or by MD only are compared in Figs. 1, 2. The Kirkwood radial distribution function (RDF) is presented in Fig. 1. It shows that the RDFs obtained by both approaches are exactly the same at all temperatures. A similar comparison for the mean-square particle displacement (MSD) demonstrates that the particle dynamics does not depend on the equilibration method for $T>T_{\mathrm{g}}$ (cf. Fig. 2). At a lower temperature $(T=0.24<T \mathrm{~g})$ a small difference is visible at long times. It signals that the standard slow cooling via MD is not sufficient to fully equilibrate the system for $T=0.24$ as the total tempering time is too small compared to the relaxation time $\tau_{\alpha}$. Note however that this regime $\left(T<T_{\mathrm{g}}\right)$ is not considered in the present paper. 


\section{SIMULATION RESULTS}

\section{A. Structure factor}

The dynamical structure factor is

$$
S(\underline{q}, t)=\frac{1}{N} \sum_{l, k=1}^{N}\left\langle e^{\left.\mathrm{i} \underline{q} \cdot\left(\underline{r}_{l}\left(t+t^{\prime}\right)-\underline{r}_{k}\left(t^{\prime}\right)\right)\right\rangle}\right.
$$

where $\underline{r}_{l}(t)$ is position of particle $l$ at time $t$, and $\langle\ldots\rangle$ means ensemble averaging. As the system we consider is isotropic, the structure factor depends only on $q=|\underline{q}|: S(\underline{q}, t)=S(q, t)$. This factor can be also defined as a correlation function of concentration fluctuations:

$$
S(q, t)=\frac{V}{c_{0}}\left\langle c_{\underline{q}}\left(t+t^{\prime}\right) c_{\underline{q}}^{*}\left(t^{\prime}\right)\right\rangle
$$

where averaging is taken over the equilibrium ensemble, $V$ is $d$-dimensional system volume,

$$
c_{\underline{q}}(t)=\int c(\underline{r}, t) e^{-\mathrm{i} \underline{q} \cdot \underline{r}} \mathrm{~d}^{d} r / V=\frac{1}{V} \sum_{k} e^{-\mathrm{i} \underline{q} \cdot \underline{r}_{k}(t)}
$$

and $c(\underline{r}, t)$ is the local concentration at time $t$ and position $\underline{r}$.

The static structure factor $S(q) \equiv S(q, 0)$ for our $2 \mathrm{D}$ system is shown for several temperatures above $T_{\mathrm{g}}$ in Fig. $3(\mathrm{a})$. Its behavior is typical for glass-forming liquids: $S(q)$ shows a strong peak at $q=q_{\max } \approx 2 \pi / \bar{\sigma}$ corresponding to the mean particle size. The behavior of $S(q)$ at low $q$ is highlighted in Fig. 3(b). A smooth dependence of $S(q)$ on $q^{2}$ is obvious; $S(q)$ is nearly linear in $q^{2}$ for $q \lesssim 1$.

Eq. 2 is not applicable exactly at $q=0$ (for a system with fixed number of particles). In this case we define $S(0) \equiv \lim _{q \rightarrow 0} S(q)$. $S(0)$ can be obtained by linear extrapolation indicated in Fig. 3(b). The minimal $q$ for which $S(q)$ can be calculated using eq. 2 with $\mathrm{PBC}$ is $q_{\min }=2 \pi / L$. For all $T$ 's considered in this study the relative deviation $S\left(q_{\min }\right) / S(0)-1$ is below $4 \cdot 10^{-4}$, which is smaller than the statistical error on $S\left(q_{\text {min }}\right)$. The difference between the extrapolated $S(0)$ and $S\left(q_{\mathrm{min}}\right)$ is therefore totally negligible; it is disregarded in what follows.

By contrast, the $q$-dependence of $S(q, t)$ is very important also for low $q$ as discussed below. [24]

\section{B. Computational results on dynamical correlations}

Let us first consider the specific heat per particle, $c_{v}=\left.\frac{1}{N} \frac{\partial E}{\partial T}\right|_{V, N}$. Its time-dependent generalization can be defined via the energy $(E)$ response to a small instant $T$-jump (from $T-\delta T$ to $T$ at $t=0$ ):

$$
c_{v}(t)=\frac{\langle E(t)-E(0)\rangle}{N \delta T}
$$

The static (equilibrium) heat capacity $c_{v}$ equals to $c_{v}(\infty)$. By virtue of the fluctuation-dissipation theorem (FDT) the response function $c_{v}(t)$ is related to the energy correlation function $C_{E}(t)=\left\langle\Delta E\left(t+t^{\prime}\right) \Delta E\left(t^{\prime}\right)\right\rangle$, where $\Delta E=E-\langle E\rangle$ and $\langle\ldots\rangle$ denotes the canonical equilibrium average (averaging over $t^{\prime}$ is assumed as well):

$$
c_{v}(t)=\frac{1}{N T^{2}}\left[C_{E}(0)-C_{E}(t)\right]
$$

For ergodic systems the function $C_{E}(t) \rightarrow 0$ at $t \rightarrow \infty$, so eq. 6 turns into the classical relation $c_{v}(\infty)=$ $\left\langle(\Delta E)^{2}\right\rangle /\left(N T^{2}\right)$. The time-dependent heat capacity $c_{v}(t)$ is thus fully defined by $C_{E}(t)$ which was recorded at different $T$ 's for $m=50 \div 100$ independent trajectories. The results for $\Delta c_{v}(t)=c_{v}(\infty)-c_{v}(t)$ at $T>T_{\mathrm{g}}$ are shown in Fig. 4. Its relaxation for short times, $t<\tau_{\alpha}$ (cf. Fig. 4(a)), involves a sharp increase, then decrease with further overshoots. This behavior is qualitatively consistent with the frequency dependence of $c_{v}$ above $T_{\mathrm{g}}$ revealed in a simulation study of viscous silica [5]. One can observe however a new feature at $t \gtrsim \tau_{\alpha}$ : a weak long-time tail emerged already well above $T_{\mathrm{g}}$, at $T=0.45$ (cf. Fig. $4(\mathrm{~b})$ ). It appears that the tail follows a power law scaling $\Delta c_{v} \propto 1 / t$ (cf. the dashed lines in Fig. 4(b)). The power-law tail gets stronger as $T$ decreases down to $T=0.325$ (which is still significantly above $T_{\mathrm{g}} \approx 0.26$ ). Remarkably, the terminal time-scale of the tail is much longer (by a factor $\left.\sim 100\right)$ than 
the structural time $\tau_{\alpha}$ defined as the relaxation time of the shear stress (cf. Fig. 5(a)) or of the coherent intermediate scattering function $\phi_{q}(t)=S(q, t) / S(q)$ for $q=q_{\max }$ at the peak of $S(q)$ (cf. Fig. 5(b)). The heat capacity $c_{v}(t)$ thus still increases for $t \gg \tau_{\alpha}$.

To verify the generality of this long-time behavior we performed MD simulations of a rather different glass-forming system - a binary mixture in 3 dimensions at $T$ well above the glass transition - to find a similar power-law tail for $\Delta c_{v}(t)$ (cf. Fig. 8 and the Appendix).

Fig. 6 presents $\phi_{q}(t)$ for the lowest wave-vector $q=q_{\min }=2 \pi / L$ at different $T>T_{\mathrm{g}}$. A pronounced long-time shoulder is visible at $T=0.5$; it develops into a quasi-plateau persisting for $t \gtrsim 1000 \tau_{\alpha}$ in the $T$-range between $T=0.4$ and 0.3. Finally, Fig. 7 shows the relative deviation from the CE, eq. 1 , defined as $\delta=1-S_{K} / S_{0}$, where $S_{K} \equiv c_{0} T / K_{T}[30]$ and $S_{0}=S\left(q_{\min }, 0\right)$ is nearly equal to $S(0)$ obtained by extrapolation of $S(q)$ to $q=0$ (cf. the previous section). One can observe that $\delta$ always exceeds $70 \%$ meaning that eq. 1 is not satisfied. A significant difference between $S(0) /\left(c_{0} T\right)$ and the compressibility $1 / K_{T}$ was also discussed for jammed packings of size-dispersed hard disks and spheres [18], and in dynamic light scattering of colloidal dispersions [34]. In both cases the difference was shown to originate from polydispersity effects. These findings support our view that the anomalous behaviors reported in Figs. 4, 6, 7 and 8 are also related to multi-component nature of the studied systems. This idea is clarified below.

\section{THEORY FOR 2-STEP RELAXATION OF COHERENT SCATTERING FUNCTION}

\section{A. Simple model}

The polydispersity effects for the dynamical structure factor are quantified below. We first outline a general theoretical approach and then combine it with a simple heuristic model to explain our simulation results.

The dynamical structure factor is defined in eq. 3 as a correlation function of concentration waves. According to the FDT $[3,29,33]$ the dynamical factor is related to the response function $R(q, t)$ defining the concentration wave $c_{\underline{q}}(t)$ induced by a weak external potential field $U(\underline{r}, t)=U_{0} e^{\mathrm{i} \underline{q} \cdot \underline{r}}$ applied to the system at $t>0$ :

$$
\left\langle c_{\underline{q}}(t)\right\rangle=-R(q, t) U_{0} / T
$$

where obviously $R(q, 0)=0$. The FDT relation is

$$
S(q, t)=c_{0}^{-1}[R(q, \infty)-R(q, t)]
$$

It implies that $S(q, 0)$ is defined by the static response $R(q, \infty)$.

Let us now focus on the low $q$ regime $(q \bar{\sigma} \ll 1)$, including, in particular, $q=q_{\min }$. The field $U$ produces the volume force $-c \nabla U$ which generates a hydrodynamic flow leading to the pressure $\left(\delta p=p_{q}\right)$ and concentration $\left(\delta c=c_{q}\right)$ waves. The mechanical balance for $t \gg \tau_{\alpha}$ then demands $\delta p \simeq-c U$ with $\delta p \simeq K_{T} \delta c / c_{0}$, hence $R(q, t) \simeq \frac{T c_{0}^{2}}{K_{T}}$ leading to the classical result for monodisperse systems, $S_{0}=S(q, 0) \simeq T c_{0} / K_{T}$ (for $q \bar{\sigma} \ll 1$ ). In the general (polydisperse) case the considered mechanism implies that the colloidal composition stays unchanged for each element of the system. This is valid for the first (fast) stage of concentration relaxation which serves to establish the local mechanical balance $(\nabla p+c \nabla U=0)$. However, later on the concentration wave amplitude (and therefore $R(q, t))$ still increases further due to an exchange between small and large particles by their slow mutual diffusion ('slow' stage). The amplitude of this increase can be deduced from a simple model assuming that the free energy of interactions between the particles depends primarily on the local volume concentration, $\phi(r)=\sum_{i} v_{i} c_{i}(r)$, where $v_{i}=\sigma_{i}^{d}$ [37]. Then, the total free energy density is (position $r$ is omitted for $\phi(r)$ and $c_{i}(r)$ ):

$$
f=\frac{1}{\bar{v}} f^{*}(\phi, T)+T \sum_{i=1}^{n} c_{i} \ln c_{i},
$$

where the first term is due to particle interactions, $\bar{v} \equiv \overline{\sigma^{d}}$, and the second term in the r.h.s. accounts for the entropy of mixing. The assumption to express the excess part $f^{*}$ in terms of $\phi$ is backed by the success of similar approximate expressions employed to explore phase equilibria in polydisperse systems [35]. The crucial role of the local volume fraction for jammed polydisperse systems was also highlighted in Ref. [18]. As we focus here on the linear response, we may expand $f$ for small deviations $\delta c_{i}=c_{i}-c_{i}^{(0)}$ from the equilibrium state, and keep only the quadratic terms (the linear term is irrelevant as its volume integral is const $=0$ ):

$$
\delta f / T \simeq v^{*} \delta \phi^{2} /\left(2 \bar{v}^{2}\right)+\sum_{i}\left(\delta c_{i}\right)^{2} /\left(2 c_{i}\right)
$$


where $\delta \phi=\sum_{i} v_{i} \delta c_{i}$ and the volume $v^{*}$ is defined by $T v^{*} / \bar{v}=\partial^{2} f^{*} / \partial \phi^{2}$ at $\phi=\phi_{0}=\sum_{i} v_{i} c_{i}^{(0)}$.

During the fast mechanical stage a fluid element deforms as a whole, keeping the same composition: $\delta c_{i} / c_{i}=\delta c / c_{0}$, where $\delta c=\sum_{i} \delta c_{i}$. Using eq. 9 with this condition leads to

$$
\delta f \simeq K_{T}(\delta c)^{2} /\left(2 c_{0}^{2}\right), \quad K_{T}=T c_{0}\left(c_{0} v^{*}+1\right)
$$

Therefore the plateau response (for $t \gg \tau_{\alpha}$ and after the fast mechanical relaxation stage) is $R_{p l} \simeq \frac{T c_{0}^{2}}{K_{T}}=\frac{c_{0}}{c_{0} v^{*}+1}$. To get the terminal amplitude $R_{\infty}=\lim _{q \rightarrow 0} \lim _{t \rightarrow \infty} R(q, t)=c_{0} S(0)$ (recall that $R(q, 0)=0$ ) we have to allow for composition variations. This is achieved by the minimization of $f$, eq. 9 , with respect to $\delta c_{i}$ with the only side condition $\sum_{i} \delta c_{i}=\delta c$. The result is: $\delta f=K^{*}(\delta c)^{2} /\left(2 c_{0}^{2}\right)$ with [36]

$$
K^{*}=T c_{0}\left[Z\left(1-S_{K}\right)+1\right] /\left[Z\left(1-S_{K}\right)+S_{K}\right]
$$

where $S_{K}=c_{0} T / K_{T}$ and

$$
Z=\sum_{i} \frac{N_{i}}{N}\left(1-v_{i} / \bar{v}\right)^{2}=\overline{v^{2}} / \bar{v}^{2}-1 \stackrel{d=2}{=} 4 \delta_{p}\left(1+\delta_{p} / 5\right) /\left(1+\delta_{p}\right)^{2}
$$

Therefore the terminal response is given by $R_{\infty}=T c_{0}^{2} / K^{*}$. On this basis we get the structure factor at vanishing $q: S(0)=R_{\infty} / c_{0}=S_{K *}$, where $S_{K *} \equiv c_{0} T / K^{*}$. It is thus predicted that $S(q, t)$ relaxes from the initial value $S_{0} \simeq S(0)=S_{K *}$ (which is close to the static structure factor at $q \rightarrow 0, S(0)$ ) to the intermediate plateau at $S_{p l}=\left(R_{\infty}-R_{p l}\right) / c_{0}$,

$$
S_{p l}=S_{K *}-S_{K}=\frac{Z\left(1-S_{K}\right)^{2}}{1+Z\left(1-S_{K}\right)}
$$

The amplitude of this decrease is thus given by $S_{K}=c_{0} T / K_{T}$. This result resonates with the Kirkwood-Buff theory $[16,17]$ : For a polydisperse system $S(0)$ can be expressed as a sum of a compressibility contribution $\left(S_{K}\right)$ and a term related to composition fluctuations $\left(S_{p l}\right)$. Here, however, $S_{p l}$ is defined as a dynamic quantity (rather than a combination of partial structure factors $[16,17])$. Note also that our approach is akin to the analysis of DLS of polydisperse colloidal suspensions in Ref. [34].

The theoretical function $\delta=S_{p l} / S_{K *}$ is compared with the simulation data in Fig. 7. A very good agreement is obvious. The proposed simple model thus accounts very well for the composition fluctuation effects. It shows that the 'polydispersity' contribution to $S_{0}$ tends to $S_{p l} \simeq \frac{Z}{Z+1}$ in the 'incompressible' limit $K_{T} \rightarrow \infty$, so $S_{p l}$ (which is roughly proportional to the size PDI, $\delta_{p}$ ) can strongly exceed the compressibility term $S_{K}$ for however low $\delta_{p}$ (cf. eq. 11).

Furthermore, we find that the terminal relaxation of $S(q, t)$ from $S_{\mathrm{pl}}$ to 0 can be described by a single diffusion constant equal to the mean self-diffusion coefficient $D_{\mathrm{s}}$ deduced from the mean-square displacements (MSD) averaged over all particles:

$$
S(q, t) \simeq\left(S_{0}-S_{K}\right) \exp \left(-q^{2} D_{s} t\right), \quad t \gg \tau_{\alpha}
$$

where $S_{0}=S(q, 0)$ and $q \bar{\sigma} \ll 1$, as noted before. The theoretical curves (dashed) are shown in Fig. 6 for $t>100$. A very good agreement with the MD data is obvious. Thus, it appears that the collective interdiffusion coefficient governing the concentration relaxation for $t \gg \tau_{\alpha}$ is close to the average self-diffusion coefficient, which means that the so-called Vineyard approximation [3] works very well here. The generality of this result for weak polydispersity is demonstrated in the next section. The importance of interdiffusion processes for glass-forming systems with size polydispersity was discussed qualitatively in Refs. [21,34] and for binary mixtures in the framework of mode-coupling theory in Ref. [20]. The theory for binary mixtures yields an expression analogous to eq. (10) with an amplitude given by the Kirkwood-Buff result for $S_{0}-S_{K}[17]$ and a relaxation rate determined by the interdiffusion coefficient (cf. eq. (10b) of Ref. [20]).

\section{B. General theory for low PDI}

In this section we obtain the long-time dependence of $S(q, t)$ up to the leading order in the deviations of $\sigma_{i}$ from the average $\bar{\sigma}$. Our analysis is akin to, but is different from the perturbative approach proposed in Ref. [18] (as only static quantities were considered there).

The argument given below eq. 7 in the previous section shows that the scattering function $S(q, t)$ (defined in eq. 3 ) relaxes in 2 stages: (i) fast compression stage, (ii) slow compositional stage. A mechanical balance is established after 
the first stage, at $t \gg \tau_{\alpha}$ : the pressure becomes nearly uniform, $p(\underline{r}) \simeq p_{0}=$ const. For monodisperse systems it also leads to complete relaxation of concentration fluctuations, i.e. $c(\underline{r})=$ const, since $p(\underline{r})$ is a function solely of $c(\underline{r})$ and $T$. (Coarse-grained functions $c(\underline{r}), p(\underline{r})$ are considered here with coarsening length much longer than particle size $\sigma$, but much smaller than $1 / q$.) However, the situation is different in a polydisperse system where pressure depends also on the local composition, that is, on the partial concentrations $c_{i}(\underline{r})$. [37]

In the general case the pressure is a function of all partial $c_{i}$ :

$$
p=p\left(c_{1}, c_{2}, . . c_{n}\right)
$$

The space position $\underline{r}$ is omitted here: it is implied that $p=p(\underline{r}), c_{1}=c_{1}(\underline{r})$, etc.; the temperature variable $T$ is omitted as well. Dealing with the function of many variables in eq. 14 is a hard problem. Fortunately, however, the things can be simplified in the case of a weak polydispersity.

It is useful to start with the reference monodisperse system involving particles of the same size $\sigma_{\text {ref }}=\bar{\sigma}(\bar{\sigma}=$ $\sum_{i} N_{i} \sigma_{i} / N$ is the mean size for the original polydisperse system), with pressure $p=p_{\text {ref }}(c)$, where $c=\sum_{i} c_{i}$. It is clear that the effect of a small deviation of a particle size from $\bar{\sigma}$ must be nearly proportional to $\sigma-\bar{\sigma}$ :

$$
p=p_{\text {ref }}(c)+\alpha \sum_{i}\left(\sigma_{i}-\bar{\sigma}\right) c_{i}+\mathcal{O}\left(\Delta^{2}\right)
$$

where $\alpha=\alpha(T)$ is a constant. The second term in the r.h.s. is linear in $\Delta=\max |\sigma / \bar{\sigma}-1|$, and $\mathcal{O}\left(\Delta^{2}\right)$ stands for the omitted quadratic (in $\Delta$ ) and higher-order terms. Solving the above equation for $c$ with $p=p_{0}$ one gets:

$$
c=c_{0}-\alpha^{\prime} \sum_{i}\left(\sigma_{i} / \bar{\sigma}-1\right) c_{i}+\mathcal{O}\left(\Delta^{2}\right)
$$

where $c_{0}$ is defined by $p_{\text {ref }}\left(c_{0}\right)=p_{0}$, and constant $\alpha^{\prime}=\bar{\sigma} \alpha\left(\frac{\partial p \text { ref }}{\partial c}\right)_{T}^{-1}$. Omitting $\mathcal{O}\left(\Delta^{2}\right)$ leads to

$$
c(\underline{r})-c_{0} \simeq-\Psi(\underline{r}) \alpha^{\prime} \Delta
$$

where $\Psi$ is the compositional order parameter

$$
\Psi(\underline{r})=\sum_{i} z_{i}\left(c_{i}(\underline{r})-c_{i}^{(0)}\right), \quad z_{i} \equiv \frac{\sigma_{i}-\bar{\sigma}}{\bar{\sigma} \Delta}
$$

and $c_{i}^{(0)}=N_{i} / V$ is the average concentration of $i$-th component. Note that $z_{i}$ is changing between -1 and 1 and that $\sum_{i} z_{i} c_{i}^{(0)}=0$ by definition of $\bar{\sigma}$. On recalling eqs. 3 and 17 , the dynamical structure factor (for the slow stage, $\left.t \gg \tau_{\alpha}\right)$ becomes

$$
S(q, t) \simeq \Delta^{2}\left(\alpha^{\prime}\right)^{2} S_{\Psi}(q, t)
$$

where

$$
S_{\Psi}(q, t)=\frac{N}{c_{0}^{2}}\left\langle\Psi_{q}(t) \Psi_{q}^{*}(0)\right\rangle
$$

Now let us consider the limit $\Delta \rightarrow 0$, where all the particles become physically the same. We can still define the order parameter $\Psi$ for this reference system treating $z_{i}$ formally as the 'color' parameter for the $i$-th group. In this case the dynamical structure factor becomes (cf. eq. 2):

$$
S(q, t) \equiv \frac{1}{N} \sum_{l, k=1}^{N}\left\langle e^{\mathrm{i} q} \cdot\left(\underline{r}_{l}(t)-\underline{r}_{k}(0)\right)\right\rangle=S_{\text {self }}(q, t)+\frac{N-1}{N} S_{\text {cross }}(q, t)
$$

where $\underline{r}_{l}(t)$ is position of particle $l$ at time $t$,

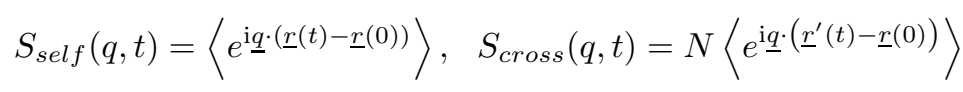

and $r(t), r^{\prime}(t)$ are positions of 2 arbitrarily chosen different particles at time $t$. Here $S_{\text {self }}$ is the self-intermediate (incoherent) scattering function, and $S_{\text {cross }}$ accounts for cross-correlations between the particles. 
Using the definition, eq. 18, we find for the monodisperse system:

$$
S_{\Psi}(q, t)=\frac{1}{N^{2}}\left(\sum_{i} N_{i} z_{i}\right)^{2} S_{\text {cross }}(q, t)+\frac{1}{N}\left(\sum_{i} N_{i} z_{i}^{2}\right)\left(S_{\text {self }}(q, t)-\frac{1}{N} S_{\text {cross }}(q, t)\right)
$$

Obviously, during the slow stage $\left(t \gg \tau_{\alpha}\right)$ the self-intermediate function relaxes by self diffusion:

$$
S_{\text {self }}(q, t) \simeq \exp \left(-q^{2} D_{\text {sref }} t\right)
$$

where $D_{s \text { ref }}$ is the self-diffusion constant of particles in the reference monodisperse system. Taking into account that $\sum_{i} N_{i} z_{i}=0$ and that $\frac{1}{N}\left(\sum_{i} N_{i} z_{i}^{2}\right)=1 / 3$ (for uniform size distribution) we get for the reference system:

$$
S_{\Psi}(q, t)=\frac{1}{3} S_{\text {self }}(q, t)=\frac{1}{3} \exp \left(-q^{2} D_{\text {sref }} t\right)
$$

Note that the term $S_{\text {cross }}(q, t) / N$ in eq. 21 was neglected since $N \gg 1$ and $S_{\text {cross }}(q, t)$ remains finite in the thermodynamic limit: $S_{\text {cross }}(q, t) \rightarrow S(q, t)-S_{\text {self }}(q, t)$ for $N \rightarrow \infty$.

Let us return to the polydisperse system with $\Delta \ll 1$. Obviously, the function $S_{\Psi}(q, t)$ now depends on $\Delta$. However, if $\Delta$ is changed to $-\Delta$, the system remains physically the same, and so does $S_{\Psi}$ (note that $\sigma_{i}-\bar{\sigma}=\bar{\sigma} z_{i} \Delta$, so reversing the sign of both $z_{i}$ and $\Delta$ does not affect either $S(q, t)$ or $\left.S_{\Psi}(q, t)\right)$. Hence there is no correction linear in $\Delta$ to either $S(q, t)$ or $S_{\Psi}(q, t)$, and the leading correction must be quadratic

$$
S_{\Psi}(q, t)=\frac{1}{3} \exp \left(-q^{2} D_{\text {sref }} t\right)+\mathcal{O}\left(\Delta^{2}\right)
$$

For the same reason the mean self-diffusion constant remains nearly equal to the reference value:

$$
D_{s}=D_{s \text { ref }}+\mathcal{O}\left(\Delta^{2}\right)
$$

Recalling eq. 19 we obtain:

$$
S(q, t) \simeq A \exp \left(-q^{2} D_{s} t\right)+\mathcal{O}\left(\Delta^{4}\right)
$$

where

$$
A=\left(\alpha^{\prime}\right)^{2} \Delta^{2} / 3+\mathcal{O}\left(\Delta^{4}\right)
$$

The above equation justifies the time-dependence of $S(q, t)$ adopted in eq. 13 . The $\Delta^{2}$ dependence of the amplitude is also in agreement with the theoretical prediction for $S_{p l}$ specified in eq. 12 . Moreover, it is easy to show that $\alpha$ is related to $p$ and $K_{T}$, leading to $\alpha^{\prime}=d\left(1-p_{0} / K_{T}\right)$. This result is based on the following covariance property: Consider the reference monodisperse system with $\sigma_{i}=\sigma_{\text {ref }}, c=c_{0}$ and pressure $p_{\text {ref }}\left(c_{0}\right)=p_{0}$. Then, if each particle is swollen in size by the factor $\lambda=1+\epsilon$ leading to $\sigma_{i}-\sigma_{\text {ref }}=\epsilon \sigma_{\text {ref }}$ (here $\sigma_{\text {ref }}$ is a constant parameter equal to $\bar{\sigma}$ before expansion) and the total concentration is changed to $c=c_{0} \lambda^{-d}$, the pressure will decrease exactly to $p=p_{0} \lambda^{-d}$ ( $\operatorname{since}$ the Kirkwood radial distribution function $g(r)$ would obviously transform to $g(r / \lambda)$ upon the expansion). Applying eq. 15 (with $\bar{\sigma}$ replaced by $\sigma_{\text {ref) }}$ ) for this transformation leads to the following relation (valid to the first order in $\epsilon \ll 1)$ :

$$
p_{0} \lambda^{-d} \simeq p_{\mathrm{ref}}\left(c_{0} \lambda^{-d}\right)+\alpha \sum_{i} \epsilon \sigma_{\mathrm{ref}} c_{i}
$$

which implies that $\alpha \bar{\sigma}=d\left(K_{T}-p_{0}\right) / c_{0}$, where $K_{T}=\left(\frac{\partial p}{\partial \ln c}\right)_{c=c_{0}, T}$. Thus, we arrive at the general result for the amplitude $A$ :

$$
S_{p l}=A=d^{2} \delta_{\mathrm{p}}\left(1-p_{0} / K_{T}\right)^{2}+\mathcal{O}\left(\delta_{\mathrm{p}}^{2}\right)
$$

where $\delta_{\mathrm{p}}=\overline{\sigma^{2}} / \bar{\sigma}^{2}-1$ is the size-polydispersity index.

The asymptotically exact expression for $S_{p l}=A$, eq. 25, gives $\delta=A /\left(A+S_{K}\right)$ plotted in Fig. 7 as a solid curve. An excellent quantitative agreement of eq. 25 with experimental data is obvious. It also agrees very well with eq. 12 .

The generalized compressibility equation therefore reads (cp. eq. 1)

$$
S(0) \simeq \frac{c_{0} T}{K_{T}}+d^{2} \delta_{\mathrm{p}}\left(1-p_{0} / K_{T}\right)^{2}
$$

which is valid for low PDI to $\mathcal{O}\left(\delta_{\mathrm{p}}^{2}\right)$. The presented argument employs the symmetry of the particle size distribution. Otherwise, it is applicable to any narrow size distribution, whether uniform or not. Note the factor $d^{2}$ in eqs. 25,26 : it shows that the structure factor $S(q, t)$ becomes very sensitive to size polydispersity for large space dimension, $d \gg 1$. 


\section{SUMMARY AND DISCUSSION}

We performed MD simulations of a $2 \mathrm{D}$ polydisperse LJ system well above $T_{\mathrm{g}}$ and found that (i) the monodisperse compressibility equation (CE), eq. 1 , is strongly violated; (ii) the dynamical structure factor $S(q, t)$ shows a two-step relaxation at low $q$, from $S_{0}$ to $S_{\mathrm{pl}}$ on the time scale $\sim 100$ (LJ units) and then from $S_{\mathrm{pl}}$ to 0 with a relaxation time much longer than $\tau_{\alpha}$ (cf. Fig. 6); (iii) the time-dependent heat capacity $c_{v}(t)$ and the related energy correlation function $C_{E}(t)$ show long-time power-law tails. The long-time power-law tail in the dynamical heat capacity gap, $\Delta c_{v}(t)=c_{v}(\infty)-c_{v}(t)$, was also identified for a 3D glass-forming mixture (cf. Appendix).

The long-time effects for $S(q, t)$ are quantitatively explained both with a simple model assuming that the excess free energy density depends only on the local volume concentration (section IV A) and with a general theory developed for a low polydispersity degree (section IV B). The theory attributes the effects to slowly relaxing composition fluctuations. The polydispersity contribution $\left(S_{\mathrm{pl}}\right)$ to $S_{0}$ is obtained in quantitative agreement with our simulation data (cf. Fig. 7 ) and exceeds $70 \%$ of $S_{0}$. Such a large deviation from eq. 1 , even for very low PDI $\left(\delta_{\mathrm{p}} \sim 1 \%\right)$, comes as a result of a competition between polydispersity and compressibility.

We obtained an asymptotically exact generalized CE (cf. eq. 26) which is valid for weakly polydisperse systems $\left(\delta_{p} \ll 1\right)$ in any spatial dimension. The polydispersity term $S_{\mathrm{pl}}$ is identified with the second term on the r.h.s. of eq. 26. Thus, the monodisperse CE can still be used once $S(0)$ is replaced with $S(0)-S_{\text {pl }}$, a result that resonates with the Kirkwood-Buff theory [18]. However, instead of determining $S_{\mathrm{pl}}$ from the matrix of static partial structure factors [18], we show that $S_{\mathrm{pl}}$ can be obtained from the relaxation of collective density fluctuations.

Moreover, we established that the terminal decay of $S(q, t)$ (for $t \gg \tau_{\alpha}$ and $q \bar{\sigma} \ll 1$ ) is exponential for weak polydispersity, with a rate defined by the self-diffusion constant $D_{\mathrm{s}}$ averaged over all particles.

We therefore established that the long-time plateau of the coherent scattering function is due to a coupling between concentration and the slowly-relaxing composition fluctuations. Our idea is that the long-time tails in $\Delta c_{v}(t)$ and the energy correlation function $C_{E}(t)$ result from a similar coupling between the total energy and composition fluctuations. Both effects are therefore generally due to the polydispersity.

It is known that glassy dynamics significantly depend on the space dimension $d$ [38-40]. Some aspects of this effect are related to soft cooperative fluctuations leading to the system size $(L)$ dependence of the particle MSD which gets more pronounced for low $d$ (in particular, for $d=2$ ) $[39,40]$. Moreover, for $d=2$ the standard MSD significantly differs from its 'cage-relative' analog [39]. While these effects are generally important, our crucial point is that (as proved in section IV B) the dynamics of composition fluctuations reflected in the long-time behavior of $S(q, t)$ are universally defined by the self-intermediate scattering function associated with the standard MSD and self-diffusion constant $D_{s}$ (cf. eq. 13).

All effects discussed above can be measured experimentally and are quite generic. They must be present in all polydisperse systems, including binary mixtures. Therefore, our work raises intriguing questions on the impact of composition fluctuations (which are important also because of growing awareness that they may trigger instability to crystallization [11]) and their interplay with the glassy dynamics [20-23].

\section{Acknowledgments}

We thank E.Bartsch for helpful discussions. A grant of computer time at the HPC computing cluster of the University of Strasbourg is gratefully acknowledged. L.K. is supported by a doctoral contract from the University of Strasbourg in the framework of the IRTG "Soft Matter Science". 


\section{Appendix A: Dynamic specific heat for a 3D binary mixture}

We also examined the binary Lennard-Jones mixture of Kob and Andersen in three dimensions [41-43]. The interaction potential is given by $u_{i j}(r)=4 \varepsilon_{i j}\left[\left(\sigma_{i j} / r\right)^{12}-\left(\sigma_{i j} / r\right)^{6}\right]$ for the two particle species $i, j=\mathrm{A}, \mathrm{B}$. The potential parameters are defined as $\varepsilon_{\mathrm{BB}}=0.5 \varepsilon_{\mathrm{AA}}, \varepsilon_{\mathrm{AB}}=1.5 \varepsilon_{\mathrm{AA}}, \sigma_{\mathrm{BB}}=0.88 \sigma_{\mathrm{AA}}$ and $\sigma_{\mathrm{AB}}=0.8 \sigma_{\mathrm{AA}}$. The mixture is therefore nonadditive and also exhibits energy dispersity. The LJ potential is truncated at $2.5 \sigma_{i j}$ and shifted to zero to avoid discontinuity. All results are presented in reduced units where $\varepsilon_{\mathrm{AA}}=1, \sigma_{\mathrm{AA}}=1$, the mass $(m)$ of the particles is set to unity as is also the Boltzmann constant. The simulated system consists of $N=1000$ particles with $N_{\mathrm{A}}=800$ A-particles and $N_{\mathrm{B}}=200 \mathrm{~B}$-particles. All simulations were performed with the LAMMPS code in the NVT ensemble at constant density $\rho=N / V=1.2$. The equations of motion were integrated with the velocity-Verlet algorithm with a time step of $0.001 \tau_{\mathrm{LJ}}=\sqrt{m \sigma_{\mathrm{AA}}^{2} / \varepsilon_{\mathrm{AA}}}$. Temperature is maintained by the Nosé-Hoover thermostat.

After equilibration we calculated the energy correlation function $C_{E}(t)$ as a time average over one trajectory of total length $10^{5} \tau_{\mathrm{LJ}}$. Fig. 8 shows the results for

$$
\Delta c_{v}(t)=\frac{1}{N T^{2}} C_{E}(t)
$$

at $T=0.9$ which is well above the glass transition temperature $T_{\mathrm{g}} \approx 0.39$ (obtained for cooling rate $=10^{-5},[44]$ ). The dashed line indicates the power-law decay, $\Delta c_{v}(t) \sim 1 / t^{3 / 2}$.

[1] A. Cavagna, Phys. Rep. 476, 51 (2009).

[2] E. Donth, The Glass Transition (Springer, Berlin-Heidelberg, 2001).

[3] J. P. Hansen and I. R. McDonald, Theory of Simple Liquids (Academic Press, London, 1986).

[4] C.A.Angell, K.L.Ngai, G.B.McKenna, P.F.McMillan, S.W. Martin, J. Appl. Phys. 88, 3113 (2000).

[5] P. Scheidler, W. Kob, A. Latz, J. Horbach, and K. Binder, Phys. Rev. B 63, 104204 (2001).

[6] Y-H.Jeong, Thermochimica Acta 304/305, 67 (1997).

[7] L. S. Ornstein and F. Zernike. Physik. Z. 19, 134 (1918).

[8] Note that $S(0)$ is related to fluctuations of the total number $N$ of particles in the grand-canonical ensemble: $S(0)=$ $(\delta N)^{2} /\langle N\rangle$, where $(\delta N)^{2} \equiv\left\langle N^{2}\right\rangle-\langle N\rangle^{2}$ is the variance of $N$. This relation remains valid also for polydisperse systems.

[9] F.H.Stillinger, P.G.Debenedetti, S.Sastry, J.Chem.Phys. 109, 3983 (1998).

[10] T.K.Haxton, Phys.Rev. E 85, 011503 (2012).

[11] T. S. Ingebrigtsen, J. C. Dyre, T. B. Schrøder, and C. P. Royall, Phys. Rev. X 9, 031016 (2019).

[12] A.Ninarello, L.Berthier, and D.Coslovich, Phys.Rev.X 7, 021039 (2017).

[13] W. van Megen, T. C. Mortensen, S. R. Williams, J. Müller, Phys. Rev. E 58, 6073 (1998).

[14] D. El Masri, G. Brambilla, M Pierno, G. Petekidis, A. B. Schofield, L. Berthier, L. Cipelletti, J. Stat. Mech.: Theory Exp. P07015 (2009).

[15] A. Ben-Naim, Molecular Theory of Solutions (Oxford University Press, Oxford, 2006).

[16] J. G. Kirkwood and F. P. Buff. J. Chem. Phys. 19, 774, 1951.

[17] A. L. Thorneywork, S. K. Schnyder, D. G. A. L. Aarts, J. Horbach, R. Roth, and R. P. A. Dullens, Mol. Phys. 116,3245 (2018).

[18] L.Berthier, P.Chaudhuri, C.Coulais, O.Dauchot, and P.Sollich, Phys.Rev.Lett. 106, 120601 (2011).

[19] R. Kurita and E. R. Weeks, Phys. Rev. E 84, 030401 (2011).

[20] M.Fuchs and A.Latz, Physica A 201, 1 (1993).

[21] F.Weysser, A.M.Puertas, M.Fuchs, and T.Voigtmann, Phys.Rev.E 82, 011504 (2010).

[22] J. Horbach, S. K. Das, A. Griesche, M.-P. Macht, G. Frohberg, and A. Meyer, Phys. Rev. B 75, 174304 (2007).

[23] P. Kuhn, J. Horbach, F. Kargl, A. Meyer, and T. Voigtmann, Phys. Rev. B 90, 024309 (2014).

[24] As a matter of fact, the dynamical structure factor does not relax at all in the limit $q \rightarrow 0: S(0, t) \equiv \lim _{q \rightarrow 0} S(q, t)=S(0)$. This feature is related to the conserved nature of concentration fluctuations $c_{q}: \dot{c}_{q} \equiv \partial c_{q} / \partial t \rightarrow 0$ for $q \rightarrow 0$. More precisely $S(0, t)=$ const comes from eq. 3 and the following relations

$$
\left|\left\langle\dot{c}_{q}(t) \dot{c}_{q}^{*}\left(t^{\prime}\right)\right\rangle\right| \leq\left\langle\left|\dot{c}_{q}(t)\right|^{2}\right\rangle=\frac{N}{V^{2}} q^{2} \frac{T}{m_{p}}
$$

where the last equation follows from eq. 4 and the canonical distribution of particle velocities. Taking also into account the standard MSD-type relation

we get

$$
S(q, 0)-S(q, t)=\frac{V^{2}}{2 N}\left\langle\left|c_{q}(t)-c_{q}(0)\right|^{2}\right\rangle
$$

$$
0 \leq S(q, 0)-S(q, t) \leq q^{2} t^{2} \frac{T}{2 m_{p}}
$$


where the r.h.s. tends to 0 as $q \rightarrow 0$ for any $t$. The genuine limit $q \rightarrow 0$ is therefore uninteresting for the dynamics.

[25] I. Kriuchevskyi, J. P. Wittmer, H. Meyer, and J. Baschnagel, Phys. Rev. Lett. 119, 147802 (2017).

[26] S. C. Plimpton, Comput. Phys. 117, 1 (1995).

[27] A. Tanguy, J. P. Wittmer, F. Léonforte, and J.-L. Barrat, Phys. Rev. B 66, 174205 (2002).

[28] J. P. Wittmer, H. Xu, P. Polińska, F. Weysser, and J. Baschnagel, J. Chem. Phys. 138, 12 A533 (2013).

[29] C. Ruscher, A. N. Semenov, J. Baschnagel, and J. Farago, J.Chem.Phys. 146, 144502 (2017).

[30] The compression modulus $K_{T}$ was determined by two methods: (i) by volume fluctuations in the NPT ensemble, $K_{T}=$ $T V /\left\langle(\Delta V)^{2}\right\rangle$, and (ii) by the stress-fluctuation formalism in the NVT ensemble [28, 31]. Both methods gave the same result.

[31] J. P. Wittmer, H. Xu, P. Polińska, F. Weysser, and J. Baschnagel, J. Chem. Phys. 138, 191101 (2013).

[32] W. Kob, H. C. Andersen, PRE 51, 4626 (1995); PRE 52, 4134 (1995).

[33] L.D.Landau, E.M.Lifshitz, Statistical Physics (Pergamon Press, Oxford, 1998).

[34] P. N. Pusey, H. M. Fijnaut, A. Vrij, J. Chem. Phys. 77, 4270 (1982).

[35] P. Sollich, J. Phys.: Condens. Matter 14, R79 (2002).

[36] Eq. 10 remains valid in any dimension $d$ with $v_{i}=\sigma_{i}^{d}$.

[37] Here all the particles are separated in groups according to their size $\sigma:$ particles with $\sigma \simeq \sigma_{i}$ belong to group $i, i=1,2, . . n$. The number of components $n$ is such that $n \gg 1$, but $n \ll N$. Thus, the number of particles in each group, $N_{i}$, is large.

[38] E. Flenner, G. Szamel, Nature Comm. 6, 7392 (2015).

[39] E. Flenner, G. Szamel, PNAS 116, 2015 (2019).

[40] B. Charbonneau et al. J.Chem.Phys. 139, 164502 (2013).

[41] W. Kob and H. C. Andersen, Phys. Rev. E 51, 4626 (1995).

[42] W. Kob and H. C. Andersen, Phys. Rev. E 52, 4134 (1995).

[43] M. Nauroth and W. Kob, Phys. Rev. E 55, 657 (1997).

[44] K. Vollmayr, W. Kob, K. Binder, J.Chem.Phys. 105, 4714 (1996). 


\section{FIGURE CAPTIONS}

FIG. 1. RDF for $T=0.5,0.4,0.3,0.24$ (from bottom to top), $r$ is distance in LJ units. The curves are shifted vertically with step 0.5 for clarity. Solid lines correspond to configurations obtained by particle swap, symbols (x) - to slow cooling with MD. There is no detectable difference between RDFs for the two types of configurations (standard MD and MC with swaps).

FIG. 2. The mean-square displacement (MSD) of particles vs. time $t$ (in LJ units) for $T=0.5,0.4,0.3,0.24$ (from top to bottom) based on initial configurations obtained by slow cooling with MD (symbols, x) and by particle swap (solid curves).

FIG. 3. (a) The static structure factor $S(q)$ for the $2 \mathrm{D}$ polydisperse model at $T=0.5$ (thin curve), 0.4 (thicker curve), 0.3 (the thickest curve). (b) $S(q)$ vs. $q^{2}$ at low $q$ 's for $T=0.5,0.4,0.3$ (from top to bottom). The straight segments (dashed lines) show extrapolation to $q=0$ for $T=0.5$ and 0.3 . The vertical bar with ' $\times$ ' symbols (red online) indicates $q_{\min }=2 \pi / L$.

FIG. 4. (a) Specific heat gap, $\Delta c_{v}(t)=c_{v}(\infty)-c_{v}(t)$, at different temperatures $T=0.45,0.4,0.35,0.325$ (curves from bottom to top at $t=1$ ) for short time $t, 0<t<4\left(t\right.$ in LJ units). (b) Long-time behavior of $\Delta c_{v}(t)=c_{v}(\infty)-c_{v}(t)$ for $4<t<10^{4}$ at $T=0.45,0.4,0.35,0.325$ (from bottom to top). $\Delta c_{v}$ is scaled by the factor of 2 for $T=0.45$ and 0.4. Dashed lines: fits with a power law, $\Delta c_{v} \propto 1 / t$.

FIG. 5. (a) Relaxation modulus $G(t)$ for $T=0.45,0.4,0.35,0.325$. (b) Coherent intermediate scattering function $\phi_{q}(t)$ for the same $T$ 's at $q \approx q_{\max } \approx 6.35$ corresponding to the maximum of $S(q)$. In both cases $T$ decreases from bottom to top.

FIG. 6. Coherent intermediate scattering function $\phi_{q}(t)=S(q, t) / S(q)$ at $q=q_{\text {min }}=2 \pi / L(L$ is the linear dimension of the simulation box). Dashed lines show theoretical prediction, eq. 13 , for the slow stage. $T=0.5,0.4,0.35$, $0.325,0.3$ (from bottom to top).

FIG. 7. $T$-dependence of $\delta=S_{p l} / S_{0}$ with (i) simulation data for $S_{0}$ and $S_{p l}=S_{0}-S_{K}$ (black crosses 'x'), (ii) theory with $S_{0}=S_{K *}=c T / K^{*}$ and $S_{p l}$ defined in eq. 12 (dashed blue curve), (iii) asymptotically exact theory with $S_{p l}=A$ defined in eq. 25 (with $d=2$ ) and $S_{0}=A+S_{K}$ (solid red curve). In all the cases $S_{K}=c_{0} T / K_{T}$ with $K_{T}$ obtained by simulations [30]. The vertical dotted line indicates $T_{\mathrm{g}}$.

FIG. 8. Log- $\log$ plot of $\Delta c_{v}(t)$ versus $t$ (given in units of $\tau_{\mathrm{LJ}}$ ) for the 3D binary mixture at $T=0.9$ (symbols). The dashed line indicates the power law $\Delta c_{v}(t) \propto 1 / t^{3 / 2}$. 


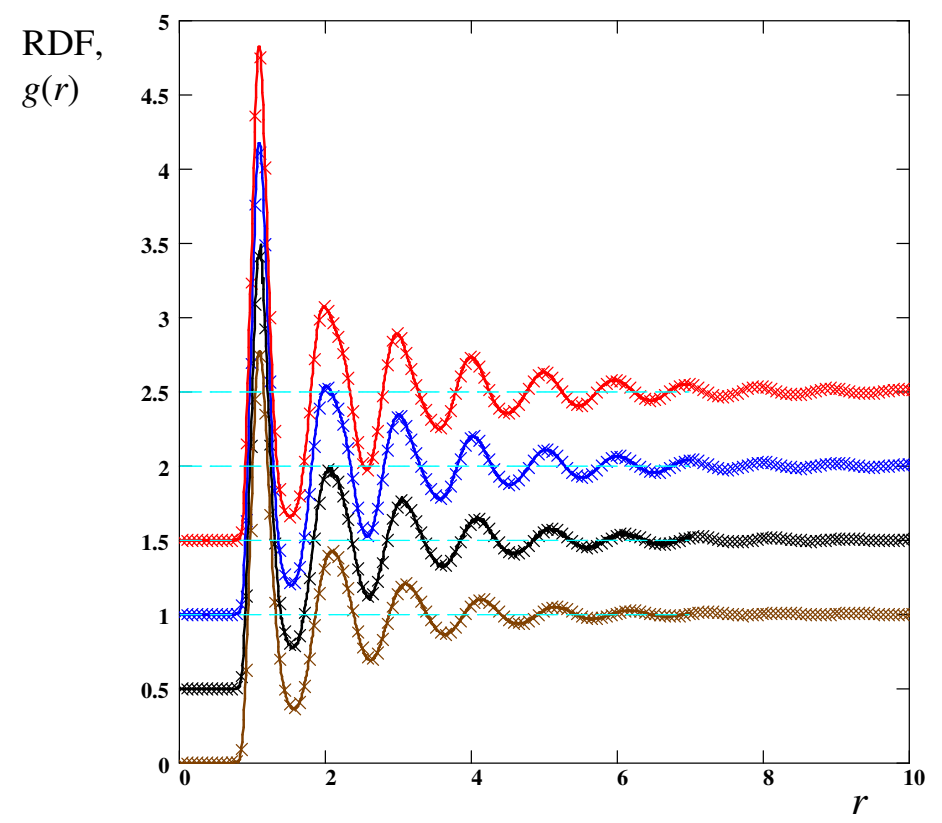

Fig. 1

Figure 1 


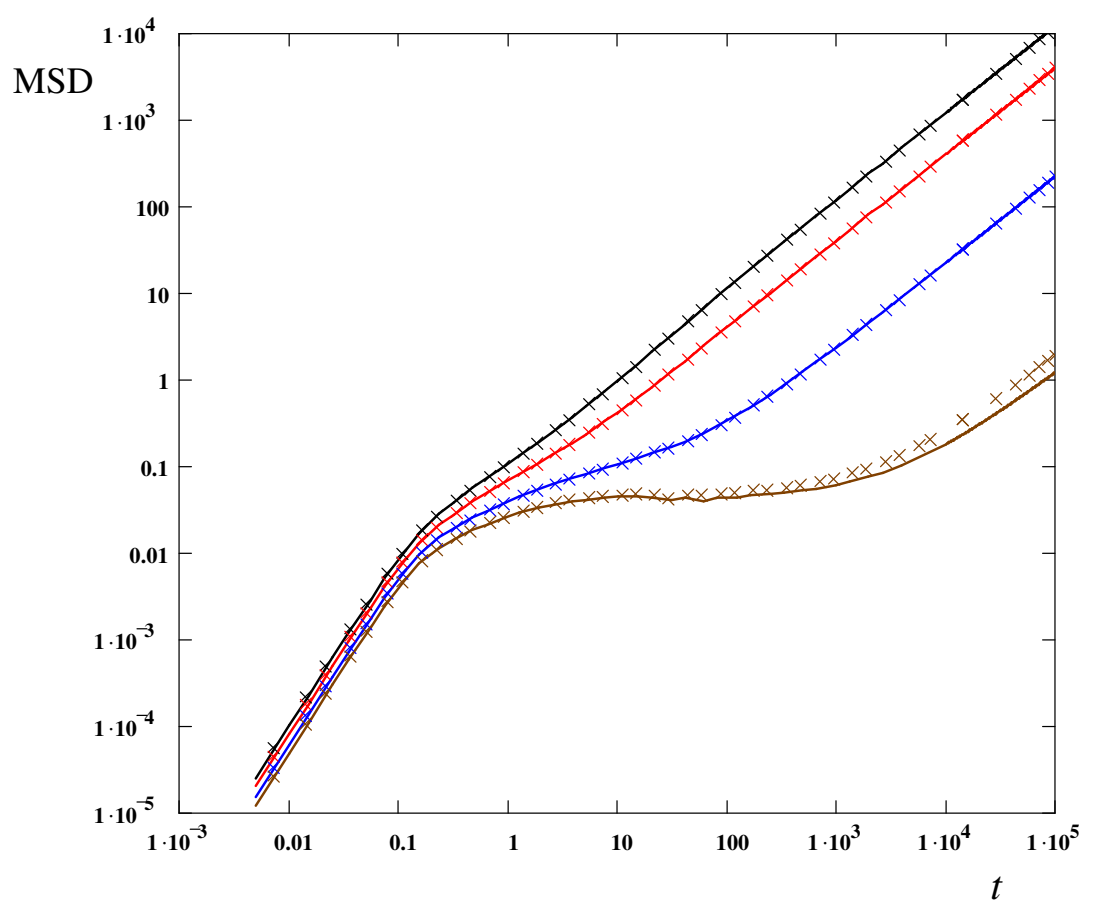

Fig. 2

Figure 2 


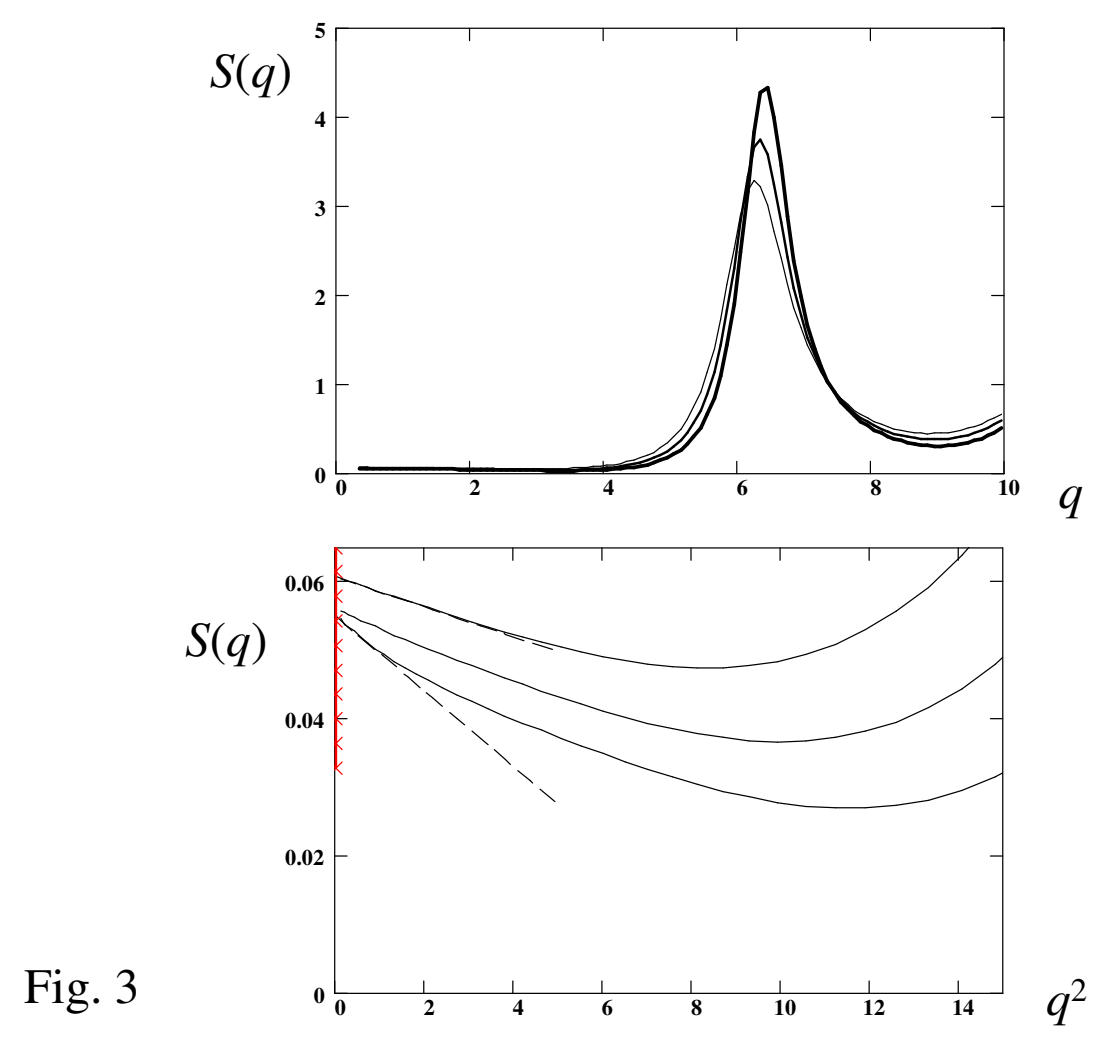

(a)

(b)

Figure 3 


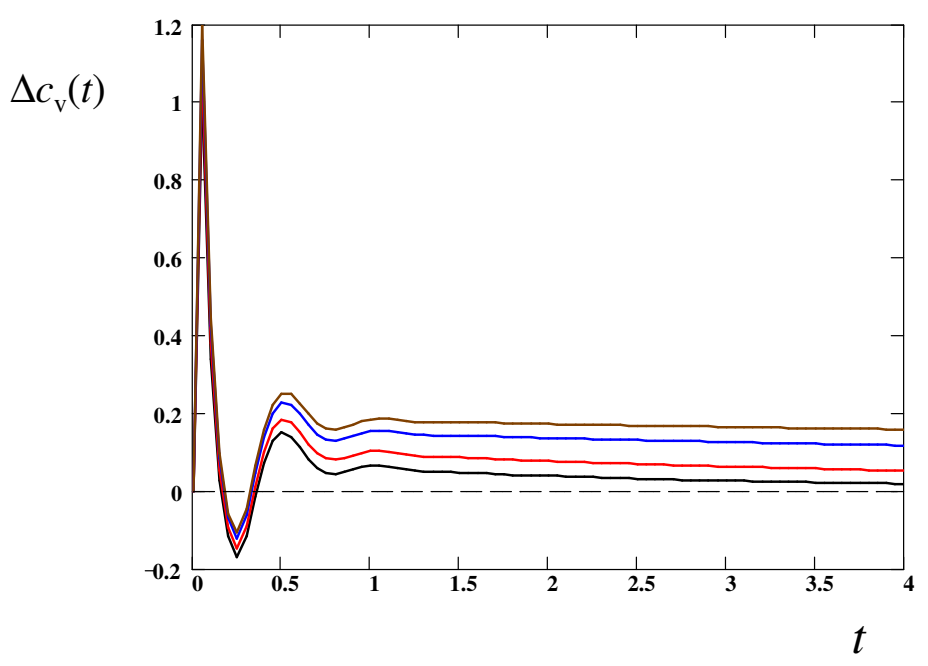

(a)

Fig. 4

Figure 4a 


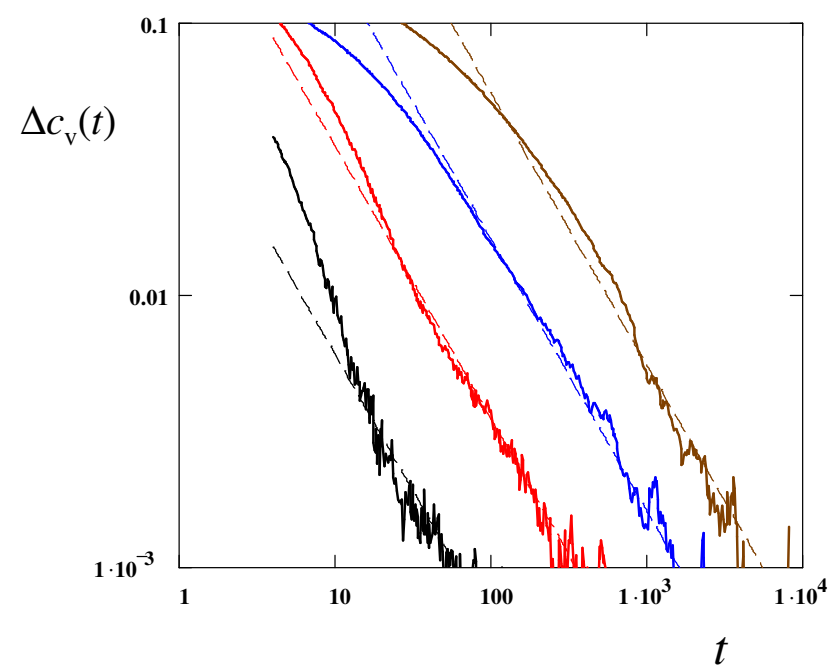

(b)

Fig. 4

Figure $4 b$ 


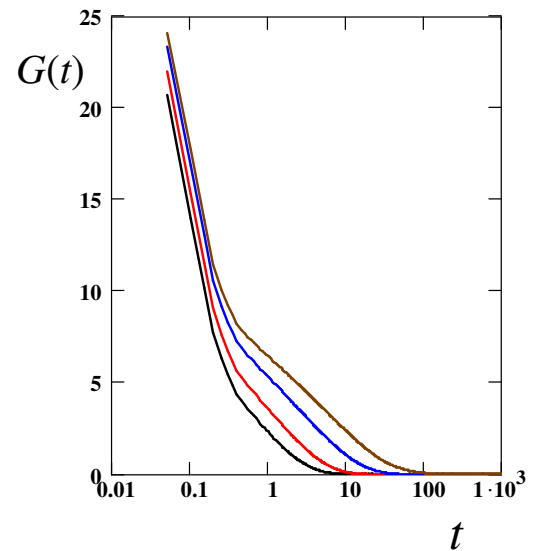

(a)

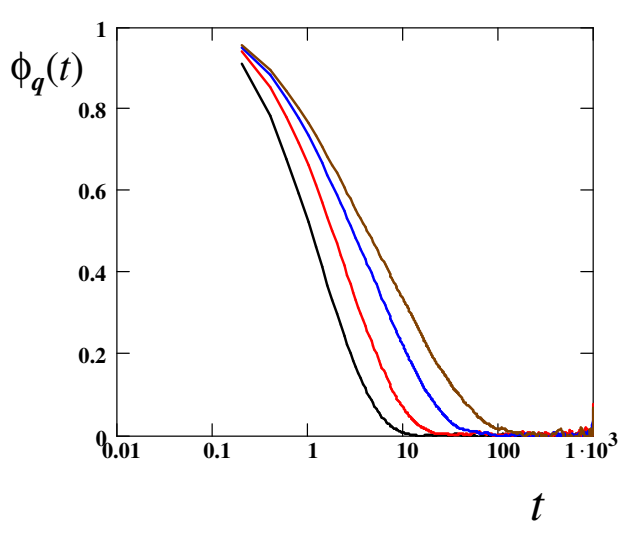

(b)

Fig. 5

Figure 5 


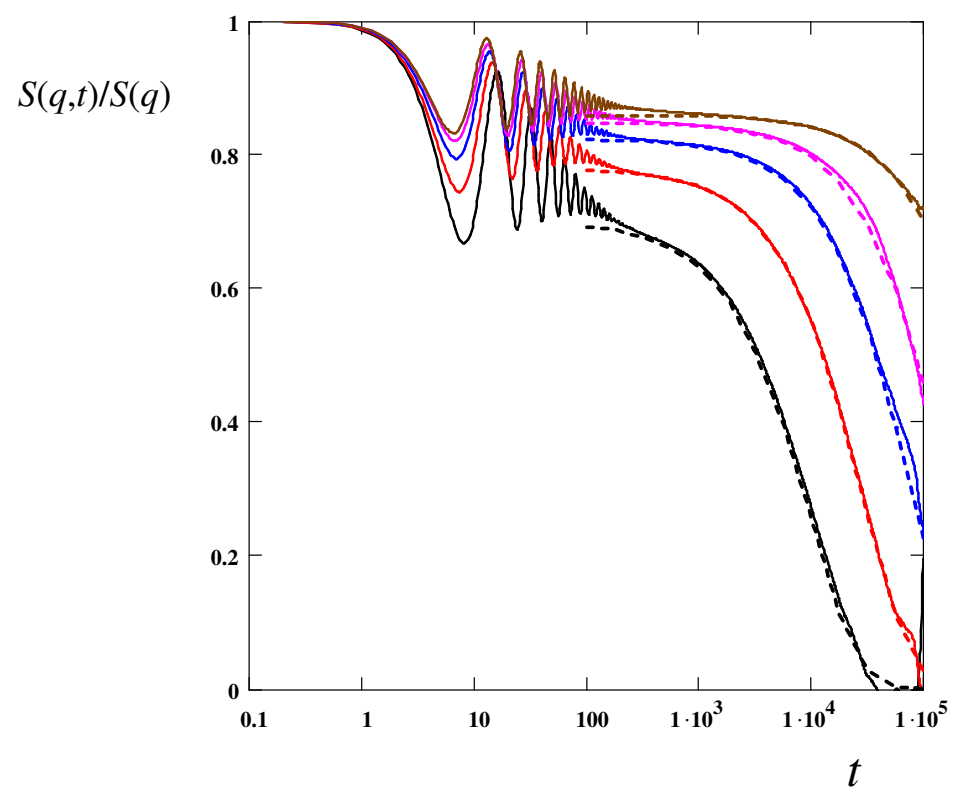

Fig. 6 .

Figure 6 


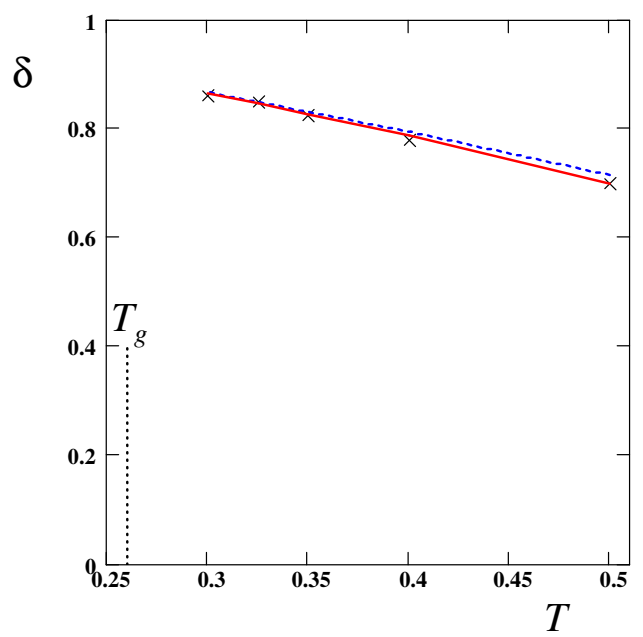

Fig. 7.

Figure 7

25Sep2020 


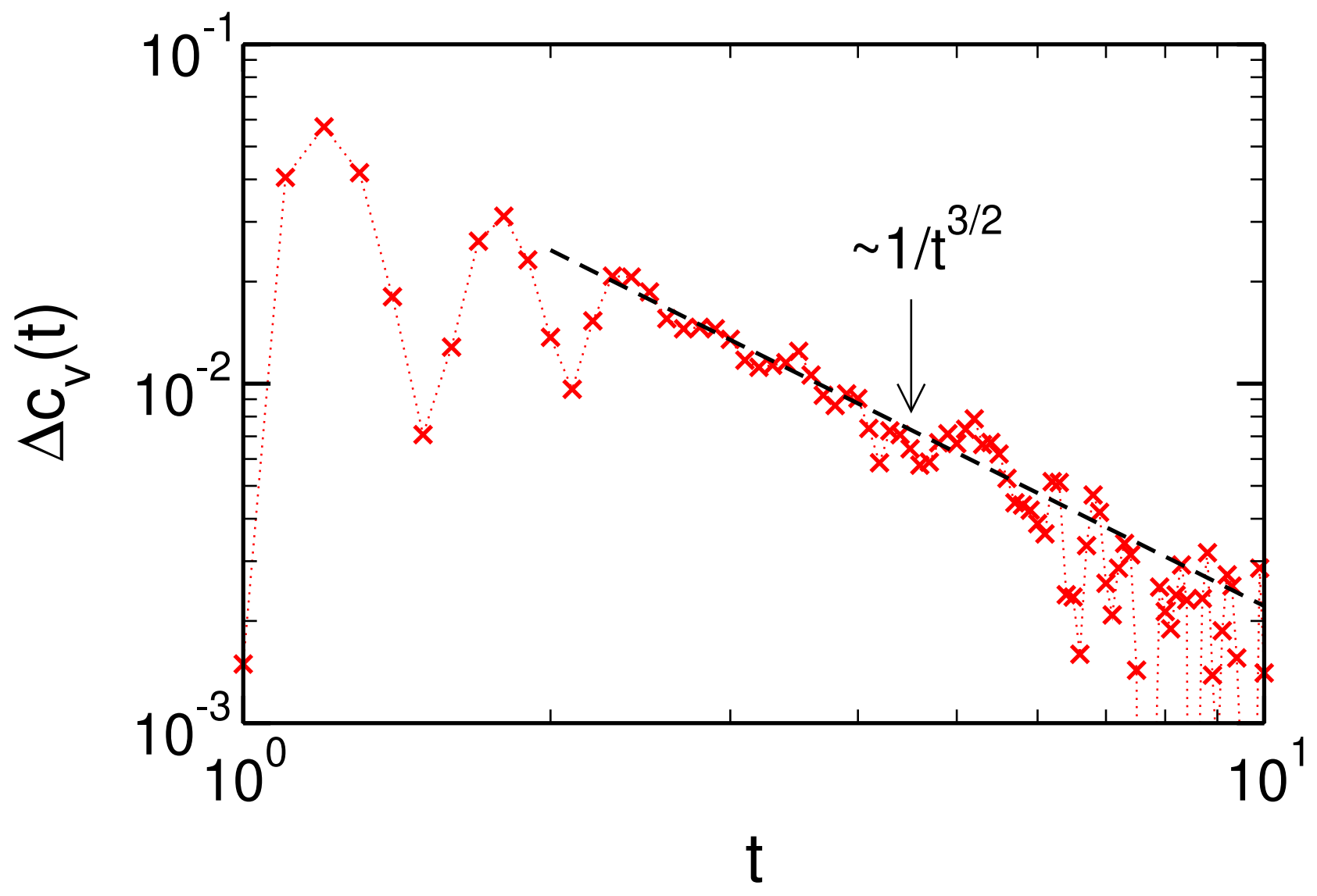

Figure 8 